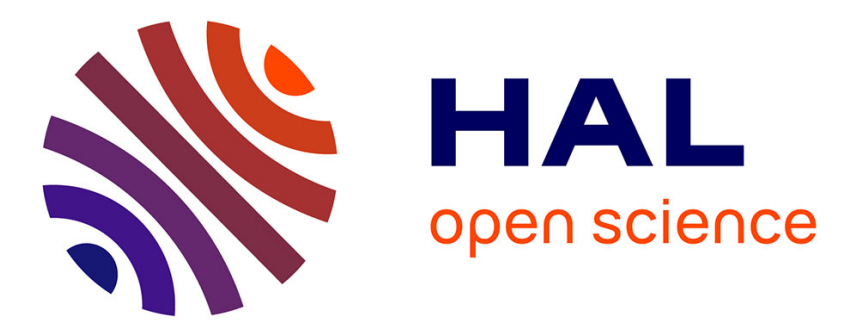

\title{
Modelling Communication Based Train control system for dependability analysis of the LTE Communication network in train control application
}

Khanh Nguyen, Julie Beugin, Marion Berbineau, Mohamed Kassab

\section{- To cite this version:}

Khanh Nguyen, Julie Beugin, Marion Berbineau, Mohamed Kassab. Modelling Communication Based Train control system for dependability analysis of the LTE Communication network in train control application. EMS2014, European Modelling Symposium, Oct 2014, Pise, Italy. 6p. hal-01073377

\section{HAL Id: hal-01073377 \\ https://hal.science/hal-01073377}

Submitted on 20 Feb 2015

HAL is a multi-disciplinary open access archive for the deposit and dissemination of scientific research documents, whether they are published or not. The documents may come from teaching and research institutions in France or abroad, or from public or private research centers.
L'archive ouverte pluridisciplinaire HAL, est destinée au dépôt et à la diffusion de documents scientifiques de niveau recherche, publiés ou non, émanant des établissements d'enseignement et de recherche français ou étrangers, des laboratoires publics ou privés. 


\section{Modelling Communication Based Train Control system for dependability analysis of the LTE Communication network in train control application}

\author{
Khanh Nguyen, Julie Beugin, Marion Berbineau \\ University Lille Nord De France \\ IFSTTAR - COSYS \\ Lille, France \\ Emails: \{khanh.nguyen, julie.beugin, marion.berbineau $\} @$ ifsttar.fr
}

\author{
Mohamed Kassab \\ HANA lab, ENSI \\ University of Manouba \\ Manouba, Tunisia \\ Email: mohamed.kassab@gmail.com
}

\begin{abstract}
The Communication Based Train Control (CBTC) systems today has generated immense interest in transport domain because of its ability to enhance safety and improve operational effectiveness. The current communication solutions for urban guided transport system that is based on the Wireless local area network (WLAN) becomes obsolete with a number of shortcomings in term of capacity and capability. Hence, the Long Term Evolution (LTE) is considered as a new solution for railway communication technology. Before widely deploying this new solution in railway industry, it must be evaluated and tested to ensure the desired performance level for CBTC system according to the railway safety standards. Therefore, it is necessary to propose a model of CBTC system that allows to consider the behaviours of the LTE communication network in real time. In this paper, we propose to use Petri nets for modelling CBTC system based on a LTE architecture. Using this model, preliminary dependability analysis for the data communication system based on the LTE technology is then performed.
\end{abstract}

Keywords-Petri net modelling; Dependability analysis; CBTC system; LTE technology

\section{INTRODUCTION}

Allowing less track-side equipment for continuous automatic train protection, the Communication Based Train Control (CBTC) systems offer lower maintenance costs, greater operational flexibility and enhanced safety. It is increasingly being applied in train control systems around the world. That is the reason why, numerous articles seek to model CBTC system in order to analyse and evaluate its performance. For general qualitative analysis of CBTC system, [13] presented 3-level hierarchy modelling approach:

1) functional model based on continuous data flow.

2) train behaviour using discrete state machine.

3) control action described as continuous data flow.

Using Colored Petri Net, [12] focused on modelling the Data Communication system (DCS) of the CBTC system in order to evaluate the transfer delay of messages between the trains and the zone controller (ZC). In [15], authors used stochastic automation networks for modelling CBTC system in order to examine the impact of the emergency braking deadline on the train stop probability.

However, in these above studies, the core of CBTC sytem - DCS system that is based on the WLAN network (standard
IEEE 802.11) cannot meet the user requirements when the accessibility and the productivity of transport system increase. In fact, due to lacking comprehensible QoS features (such as end-to-end resource management), its potential to support a multi-service delivery network in critical environments is limited. Another problem with WLAN is its insufficient capacity. In central stations, providing sufficient number of channels to serve all the trains is a major problem, especially when new passenger services (such as multimedia entertainment applications for passengers) are deployed. In addition, the delay of WLAN handover procedure also limit the possibility of implementation of CBTC system for high speed trains.

Because of the ability to support multi-service traffic (voice, video, data) that demands resilience or highbandwidth real-time capabilities, LTE offers an immense perspective to railway industry. In details, with a low userplane latency, LTE leads to higher efficiency in train operation system. Through fast handover and global roaming procedure, it also meets the mobility requirements of CBTC system with target up to $350 \mathrm{~km} / \mathrm{h}$. Moreover, QoS management mechanism built into LTE allows to guarantee delivery of critical traffic over multi service network. These above features offer a platform for further evolution and growth for CBTC system in the long term. [9] highlighted the benefits when deploying the LTE network for European Train Control System (ETCS). Using OPNET (Optimized Network Engineering Tool) simulation, authors proved that QoS mechanisms are efficient enough to use LTE for combining safety and non-safety applications. In this context, SYSTUF [11] - a French research project aims to demonstrate the feasibility of using a single communication technology based on LTE to simultaneously meet the performance requirements of safety-critical and non-critical applications in transport domain. [8] presented the results obtained within this project. It also focused on studying the QoS management mechanism proposed by LTE. Based on the OPNET modeller, the authors evaluated the performances offered by LTE to these applications, such as EndTo-End delay and packet loss. Therefore, they highlighted the LTE's ability to ensure an efficient service for guided 
transport application categories.

However, the increased dependency of multi-applications on this communication-based technology introduces new risks. The new solution must be evaluated and tested to ensure the desired performance level for safety application (CBTC) according to the railway safety standards [1]. In this paper, we will perform the preliminary dependability analysis for the communication LTE network in the train control application. The main difficulty of this task is to develop a model that permits to well consider the faults / errors / failures in the transmitted signals in real time. It should also help to see the impact of the communication parameters (such as loss connection rate, handover apparition rate etc) on the dependability parameters of the DCS in the train control application.

The paper is structured as follow: in Section II, we will describe the system and model formulation. The contribution, which concerns an approach for modelling and simulating the LTE-based-CBTC system, will be presented in Section III. Then, the Section IV aims to apply our model for the dependability analysis of the DCS of CBTC system. Finally, Section $\mathrm{V}$ presents the conclusion and the further research works.

\section{PROBLEM STATEMENT AND MODEL FORMULATION}

\section{A. Problem statement}

We consider an automatic metro line equipped with CBTC (Communication Based Train Control) system implemented on a LTE architecture. During the journey, the position/integrity message generated by a train is transmitted periodically to the Zone Controller (ZC) each $T_{s}$ second (s). After taking into account the position of other trains and the indications given by the railway signalling equipment (signal, track circuit for example), the ZC sends back to the train the movement authority (MA) - "permission to proceed". This task takes the time of $D_{Z C}$ (s). For all messages sent by train or by $\mathrm{ZC}$, they are considered valid at the reception if and only if their transmission times do not exceed a value $D_{o}$ (s) (obsolescence deadline for a message).

From the point of view of users, the data communication system in the CBTC application is considered as failed when the train does not receive a valid MA that corresponds to its position message sent before. Thus, the dependability parameters for the DCS in CBTC system will be evaluated at the level of a given train. In order to reach this objective, the performance parameters of the communication process between the train and the $\mathrm{ZC}$ will be examined in the next subsections.

\section{B. Model formulation for performance parameters of com- munication process between the train and the $\mathrm{ZC}$}

1) Transmission delay: Let $D_{p}$ be the transmission delay of the packet sent by the train or by the $\mathrm{ZC}$ in the normal case when the connection loss and handover do not occur.
Most of studies use an exponential distribution to represent this random variable. However the exponential distribution is only based on the average value of the variable and cannot handle its upper or lower bounds. Therefore, we propose to use the log-normal distribution to characterize $D_{p}$ because of its appropriate features. In fact, log-normal distribution aims to represent the random positive variables. Moreover, defining two parameters: (1) the mean value, $m_{p}$, and (2) the error factor (that is calculated as the ratio of the $95 \%$ to the median), $q_{p}$, it is easy to handle the mean value and the upper bound for the transmission delay. The probability density function of $D_{p}$ is given by:

$$
f_{D_{p}}=\frac{1}{D_{p} \cdot \sigma \sqrt{2 \pi}} \exp \frac{-\left[\ln \left(D_{p}\right)-\mu\right]^{2}}{2 \sigma^{2}}
$$

where $\mu, \sigma$ are calculated by the following equations ([10])

$$
\begin{array}{r}
\sigma=\frac{\ln \left(q_{p}\right)}{1.64} ; \\
\mu=\ln \left(m_{p}\right)-\frac{\sigma^{2}}{2} .
\end{array}
$$

2) Retransmission mechanism: LTE uses the mechanism HARQ type 3 for the retransmission occurring in the case of long acknowledgement delay or packet loss. In this document, we consider the Sync HARQ. The maximal retransmission number for a message is 3 times, i.e there are 4 packets (including an original packet) resent at maximum for a message sent by the train or the ZC [14], [7].

- When a message is sent, 3 other copies are created. If the transmitter $(\mathrm{Tx})$ receives $\mathrm{ACK}$ sent by the receiver $(\mathrm{Rx})$, these copies are deleted. For simplicity, it is unnecessary to consider the retransmission mechanism for ACK; and the ACK transmission delay is assumed to be constant and is called $D_{A C K}$.

- After the retransmission time, $T_{R E}$, if $\mathrm{Tx}$ does not receive yet $\mathrm{ACK}$, it sends another packet.

- Due to temporarily bad radio signal conditions, the packet loss occurs with probability $p_{E T}$.

Note that the obsolescence deadline $D_{o}$ is applied for the message including the retransmission ( 1 original version and 3 copies).

3) Connection loss: Let $D_{l}$ be the time to the connection loss, and $D_{r}$ be the duration time to reconnect. $D_{l}$ is assumed to follow the exponential distribution with a rate $\lambda_{l},[16]:$

$$
f_{D_{l}}(t)=\lambda_{l} \exp \left(-\lambda_{l} \cdot t\right)
$$

The train equipment detects this state after a timeout, $T_{d}$ (s) and then tries to establish a new connection with the failed reconnection probability, $p_{f}$. In detail, when the reconnection duration is more than $b$, the re-establishment is considered as false and then another attempt is made. The recovery time of a successful connection is assumed to follow the uniform distribution of $\operatorname{UNIF}[a, b]$. Thus, for 
$n \in\{1,2, \ldots \infty\}$, the probability density function of the duration of the re-connection is given by:

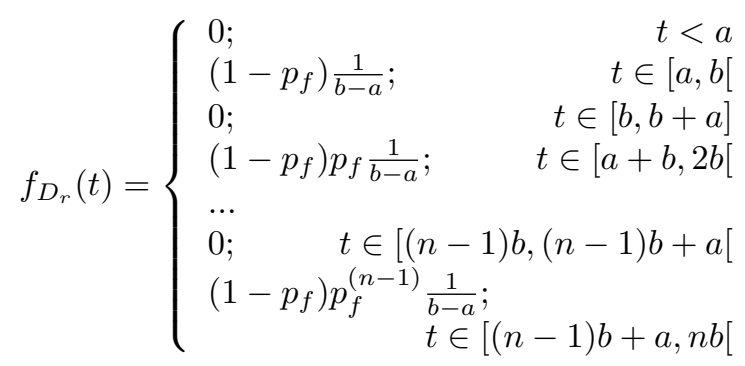

The cumulative distribution function of the reconnection duration is therefore:

$$
F_{D_{r}}(t)=\left\{\begin{array}{r}
0 \\
\sum_{i=0}^{n-1} p_{f}^{n-1} \cdot\left(1-p_{f}\right) \cdot \frac{t-(n-1) b-a}{b-a} \\
t \in[a, n b]
\end{array}\right.
$$

Note that: In reality, for safety applications, when no valid MA is received by train within a certain period, the emergency braking is triggered. However, in the framework of this article, we are only interested in the dependability of the LTE communication network and we do not consider the safety application. Then, the upper bound for the reconnection time is not defined.

4) Handover performance: Handover processes occur every time the train crosses the border between the coverage areas of two neighbouring e-nodeB. When the train is operating normally, the inter-occurrence time of handovers can be assumed to be independent and exponentially distributed with the rate $\lambda_{H o},[16]$. Then, the distribution of the time $T_{k}$ where handover occurs at the $k$-th time follows the Gamma law. Thus,

$$
F_{T_{k}}(t)=1-\sum_{j=0}^{k-1} \frac{\left(\lambda_{H o} . t\right)^{j}}{j !} \exp \left(-\lambda_{H o} t\right)
$$

The execution time of handover is assumed to follow the $\log$ normal distribution with the mean value $m_{e H}$ and the error factor of $5 \%, q_{e H}$. Let $D_{e H}$ be the handover delay, then its pdf is similarly defined as the Eq. (1).

When the packet is sent during the handover processes, its stochastic transmission delay is defined by $D_{e H}+D_{p}$.

\section{System Modelling and Simulation}

Among the modelling methods, Petri net (PN) is a graphical and mathematical modelling tool for the description of time dependent behaviours of systems ([6]) and is widely employed in dependability assessments.

In this paper, we propose to use the Petri Net module of GRIF [2] that is a software platform for determining the essential dependability parameters of a system. In details, the Petri Net module serves to model the behaviour of complex systems using stochastic Petri nets with predicates

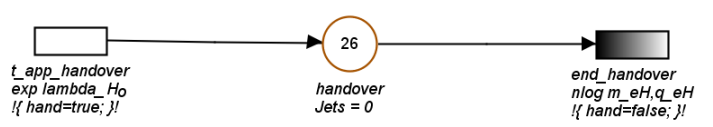

Figure 1. PN structure for the handover procedure

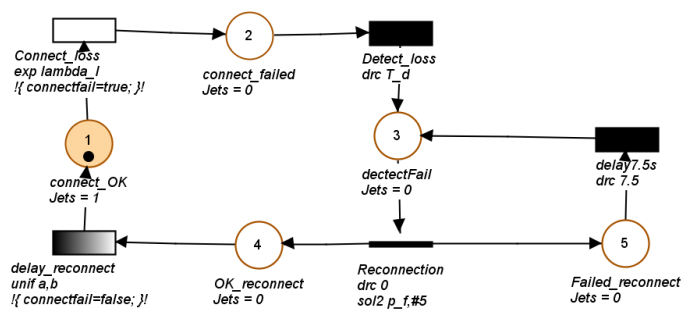

Figure 2. PN structure for the re-connection procedure

and assertions. For quantitative evaluation, it uses a highspeed Monte Carlo simulation engine (MOCA-RP).

Using the Petri net module of the GRIF platform, the CBTC system is then modelled by 4 sub-nets describing :
A. handover procedure
B. re-connection procedure
C. transmission and re-transmission procedure
D. ZC operation

The notation of PN modelling are taken from the [4] standard.

\section{A. Sub-net of the handover procedure}

In Figure 1, when the "t_app_handover" transition (that follows the exponential distribution with the rate, $\lambda_{H o}$ ) is triggered, the variable "hand" becomes true, that means the handover procedure is executed. This procedure ends when "end_handover" transition is triggered. At this moment, the the variable "hand" is set to false.

\section{B. Sub-net of the re-connection procedure}

In Figure 2, after the "Connect_loss" transition has been triggered, the variable "connectfail" becomes true. When the train detects the connection loss (i.e "Detect_loss" transition is triggered after $T_{d}$ ), the re-connection procedure described in section II-B3 is executed. For the "Reconnection" transition, only one of the downstream places ("OK_reconnect" or "Failed_reconnect") is filled after firingthe transition with its correspondent probability. In details, the token comes to the "Failed_reconnect" place with the probability, $p_{f}$. After a successful re-connection, the variable "connectfail" is set to false.

\section{Sub-net of the transmission and the re-transmission pro- cedures}

The transmission and re-transmission procedures are presented in Figure 3. In detail, every $T_{s}(\mathrm{~s})$, the "T_train_mess" transition is triggered, one packet is sent to the $\mathrm{ZC}$ and 


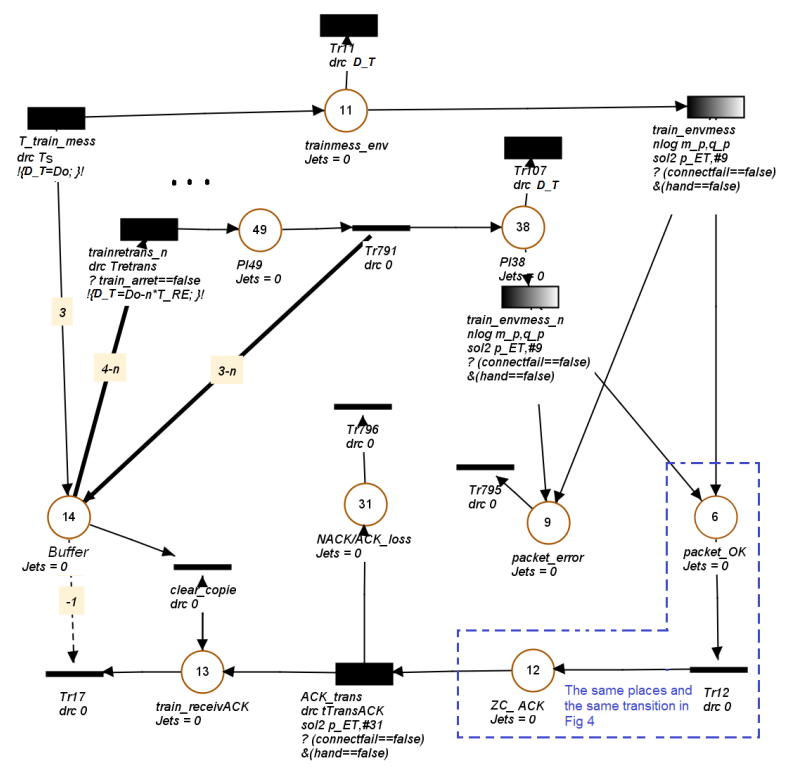

Figure 3. PN structure for the transmission and re-transmission procedures

3 other packets are simultaneously created and stored in the "Buffer" place. The deadline time - variable "D_T" is also calculated. For the first packet, "D_T" is set to the obsolescence deadline, $D_{o}$. The "train_envmess" transition represents the transmission delay for the first packet. It is triggered in the normal case when the variables "connectfail" and "hand" are false.

After triggering the "train_envmess" transition, the packet arrives at the $\mathrm{ZC}$ with the error probability $p_{E T}$. If the packet is $\mathrm{OK}$, the transition "Tr12" is triggered, the ZC sends back an ACK to train with delay "tTransACK". After triggering "ACK_Trans", if the train receives the ACK with the probability $1-p_{E T}$, it clears all of copies in the buffer by triggering the "clear_copie" transition. After the retransmission time, $T_{R E}$, if the train does not yet receive the ACK, the "trainretrans_n" transition $(n=1,2,3$, the number of retransmission) is triggered. Then, the "D_T" for the $n$-th packet is evaluate by the difference between the obsolescence deadline, $D_{o}$ and the retransmission time, $n \times T_{R E}$. The transmission procedure for the $n$-th packet is similar to the first packet, replacing the "train_envmess" by the "train_envmess_n" transition.

\section{Sub-net of ZC operation}

Figure 4 presents the model for the ZC operation: "updating MA". In details, after receiving a valid packet from the train, "Tr12" is triggered, ZC sends an ACK to the train. The ACK processing of the train was modelled in the previous section. Another token simultaneously comes to "ZC_preaction" place. Within the deadline $D_{o}$ of the train message, if the $\mathrm{ZC}$ receives another copies of this train

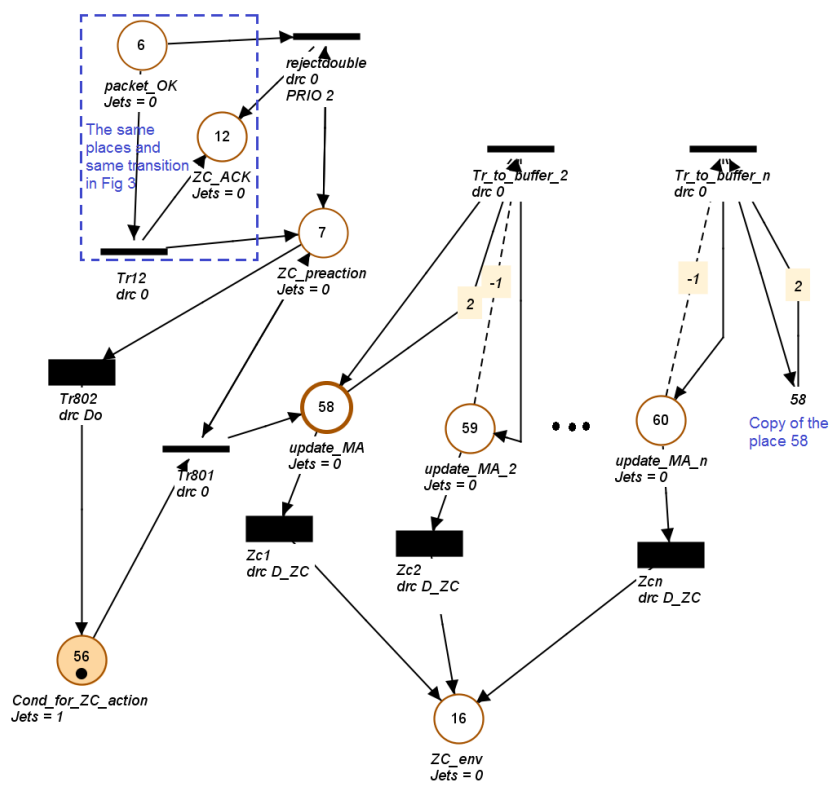

Figure 4. PN structure for $\mathrm{Zc}$ model

message, "rejectdouble" transition is then triggered, these copies are rejected.

The delay time for updating MA is $D_{Z C}$. After $D_{Z C}$ (s), the transition " $\mathrm{Zc1}$ " is then triggered, a token comes to the place "ZC_env" for starting a transmission procedure of MA from the $\mathrm{ZC}$ to the train. This procedure is modelled similarly to the transmission procedure of the train message that is presented in the previous section.

Note that when the $D_{Z C}$ is larger than the $T_{s}$, it is possible that there exists a token in the place "update_MA" that is waiting to be handled while the next message of the train (called second token) comes to the ZC. In this case, the second token is sent to the "update_MA_2" place. Similarly, if the $n$-th token comes to ZC while all $(n-1)$ places ("update_MA_1", ... "update_MA_(n-1)" places) have tokens, this $n$-th token is sent to "update_MA_n" place for handling.

\section{DePEndability ANALYSIS For CBTC SySTEM}

The information obtained during the system modelling and its simulation can lead to average values, probabilities or distributions that can serve for the dependability evaluation. Firstly, we introduce the characteristics that can be obtained and explains how they can be calculated in the subsection IV-A. Next, the results of the dependability analysis for our case study will be presented in the subsection IV-B.

\section{A. Definitions of dependability parameters}

Every $T_{s}(\mathrm{~s})$, the train sends to the $\mathrm{ZC}$ its position message. We propose to use $T_{s}$ as a time unit (TU) for evaluating the dependability parameters of DCS in the train control application. That means the DCS will be evaluated 

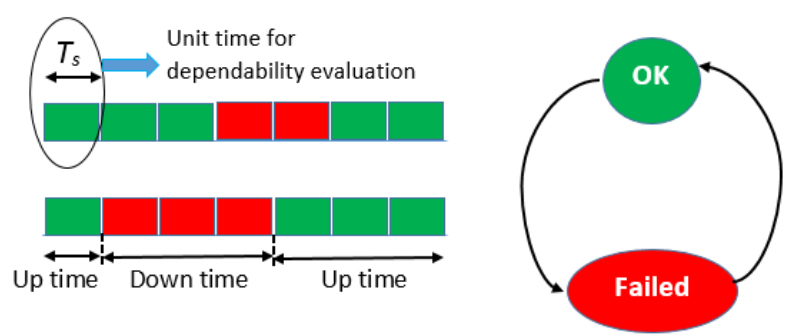

Figure 5. Examples of two scenarios constituted of successive states reached by the DCS in the train control application

as failed or not during every period of $T_{s}$ (s). In details, let $t=0$ be the moment where the first position message of the train is sent to the $\mathrm{ZC}$, then at $t=2 D_{o}+D_{Z C}$ where $D_{o}$ is the obsolescence deadline for a single way of message (from the train to the ZC for example), we will verify whether a valid MA has been received by the train or not. If not, the system is considered as failed during the first period, $\left[0, T_{s}[\right.$. Similarly, the system is considered as failed during the second period, $\left[T_{s}, 2 T_{s}\left[\right.\right.$, if at $t=T_{s}+2 D_{o}+D_{Z C}$, the train does not yet receive the MA that corresponds to the second position message. So, the down time only includes periods when the train can not receive the valid MA. The up time only includes periods when the valid MA can arrive to the train. This is illustrated in Figure 5.

Then, for the dependability analysis of the LTE communication network in the train control application, the following parameters will be evaluated:

- The MDT (Mean Down Time) that characterizes the average value of the down time, it is calculated by the average on all down time period lengths on the different scenarios.

- The MUT (Mean Up Time) that characterizes the average value of the up time. It is calculated by the average on all up time period lengths on the different scenarios.

- The MTFF (Mean Time to First Failure) that presents the average value of the first time when the train can not receive a valid MA, it is calculated by the average on all first up time period lengths on the different scenarios.

- The availability is the ability of the system to be in a state to perform the required function over a given mission time. The average availability is calculated by the average between the number of valid MA(s) received by the train and the number of position messages sent by the train on the different scenarios.

\section{B. Analysis of the case study}

In this section, we consider the following case study : Every $T_{s}=0.2(\mathrm{~s})$, the train sends a position message to the $\mathrm{ZC}$, the obsolescence deadline for a message is $D_{o}=$ 0.05 (s). The other parameters are given in the Table I
TABLE I

INPUT PARAMETERS FOR THE CASE STUDY

\begin{tabular}{|l|ccc|}
\hline Connection loss & \multicolumn{3}{|c|}{$\lambda_{f}$} \\
\cline { 2 - 4 } & $2.78 \mathrm{E}-7 / \mathrm{s}^{(1)}$ & $0.001^{(2)}$ & \\
\hline \multirow{2}{*}{$\begin{array}{l}\text { Transmission delay } \\
\text { \& ZC action }\end{array}$} & $m_{p}$ & $q_{p}$ & $D_{Z C}$ \\
\cline { 2 - 4 } Handover & $5 \mathrm{~ms}^{(3)}$ & $2.5^{(1)}$ & $1 \mathrm{~s}$ \\
& $m_{e H}$ & $q_{e H}$ & $\lambda_{H o}$ \\
\cline { 2 - 4 } & $0.08 \mathrm{~s}^{(1)}$ & $1.9^{(1)}$ & $0.03 \mathrm{~s}$ \\
\hline Retransmission & $D_{A C K}$ & $T_{r e}$ & $p_{E T}$ \\
\cline { 2 - 4 } & $4 \mathrm{~ms}^{(4)}$ & $8 \mathrm{~ms}^{(4)}$ & $0.1^{(4)}$ \\
\hline Reconnection delay & $a$ & $b$ & $T_{d}$ \\
\cline { 2 - 4 } & $0.18 \mathrm{~s}^{(6)}$ & $7.5 \mathrm{~s}^{(2)}$ & $1^{(2)}$ \\
\hline
\end{tabular}

TABLE II

DEPENDABILITY PARAMETER FOR CASE STUDY

\begin{tabular}{|c|c|c|c|}
\hline MDT & MUT & MTFF & Availability \\
\hline $1 \mathrm{TU}$ & $398.4 \mathrm{TU}$ & $649.55 \mathrm{TU}$ & $99.74 \%$ \\
$0.2 \mathrm{~s}$ & $78.96 \mathrm{~s}$ & $129.91 \mathrm{~s}$ & \\
\hline
\end{tabular}

Note that the input parameters is chosen ${ }^{(1)}$ arbitrarily but try to come close to reality, ${ }^{(2)}$ based on [16], ${ }^{(3)}$ based on [14], ${ }^{(4)}$ based on [7], ${ }^{(5)}$ based on [3], ${ }^{(6)}$ based on [5].

After performing the simulation during $1000 \mathrm{~h}$ of mission time with 100 scenarios, the results of the dependability analysis is then given in Table II. The LTE-based-DCS availability in the train control application is $99.74 \%$, i.e. among 100 messages sent, the train will receive 99.74 valid MA(s) from the ZC. On average, after every 398.4 valid MA(s) $($ MUT $=398.4 \mathrm{TU})$ received by the train from the $\mathrm{ZC}$, one MA (MDT = $1 \mathrm{TU}$ ) is considered as failed and cannot reach the train. However the first failed MA occurs after 649.55 valid MA(s) on average (MTFF $=649.55 \mathrm{TU})$. The MTFF is greater than MUT because the system has a failure probability that increases over time.

Next, Figure 6 shows the impact of the packet error probability $\left(p_{E T}\right)$, the loss connection rate $\left(\lambda_{l}\right)$ and the handover occurrence rate $\left(\lambda_{H o}\right)$ on the system availability. We find that among these parameters, the impact of $\lambda_{l}$ is non-significant. Hence, it is not necessary to improve the loss connection rate. It is better to reduce the handover occurrence rate in order to improve the system availability.

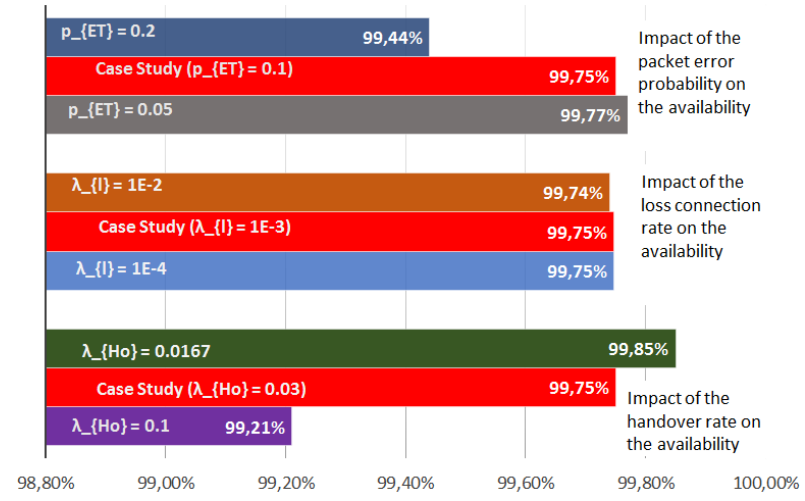

Figure 6. Impact of $p_{E T}, \lambda_{l}, \lambda_{H o}$ on the system availability 
In fact, when the mean time between handover occurrences is $60(\mathrm{~s})\left(\lambda_{H o}=0.0167\right)$, the system availability attains $99.85 \%$.

\section{CONClusion}

In this paper, using PN with assertions and predicates, we presented an approach for modelling the CBTC system, where LTE network is used for data communication between the train and the ZC. This model allows to consider the transmission procedure in real time and to take into account the failures/ errors of the communication system. It is also powerful for the dependability analysis of the CBTC system. The results highlighted the availability of the LTE-basedDCS in train control application. Moreover, for high system availability, it is not necessary to improve the loss connection rate but is better to extend the mean time between handover occurrences.

These above results that are preliminary conclusions obtained using the illustrated numerical example will be used in further work for safety analysis of CBTC system considering distance management between trains. In addition, we will examine the dependability sensitivity and study the impact of performance parameters of the communication network on the CBTC dependability parameters. On the other hand, a more efficient algorithm to improve the implementation time of Petri Net for simulation could also be developed.

\section{ACKNOWLEDGEMENT}

This work was performed within the SYSTUF project, which is subsidized by the French ministry of Industry in the framework of the AMI ITS program.

\section{REFERENCES}

[1] EN 50129, 2003. Railway applications communication, signalling and processing systems safety related electronic systems for signalling. CENELEC European standard (European Committee for Electrotechnical Standardization).
[2] GRIF, GRaphical Interface for reliability Forecasting, http://grifworkshop.com/grif/petri-module/

[3] IEEE Standard for Communications Based Train Control (CBTC) Performance and Functional Requirements, 2004.

[4] IEC 62551 2012. Analysis techniques for dependability-Petri Net techniques. Standard of the International Electrotechnical Commission.

[5] S. Mohan, R. Kappor and B. Mohanty. Latency in HSPA Data Networks, Qualcom, Tech. Rep., 2011.

[6] T. Murata. Petri Nets: Properties, Analysis and Applications. IEEE Transaction on Reliability 51 (2), 2002, pp. $541-580$.

[7] S. Kangude. Lecture: MAC - HARQ \& Scheduling. EETS 8316, Wireless Networks, SMU Engineering. Fall 2013.

[8] A. Khayat, M. Kassab, M. Berbineau, M. A. Abid \& A. Belghith LTE Based Communication System for Urban Guided-Transport: A QoS Performance Study. Communication Technologies for Vehicles. Lecture Notes in Computer Science Volume 7865, 2013, pp 197-210

[9] A. Sniady \& J. Soler. Performance of LTE in High Speed Railway Scenarios: Impact on Transfer Delay and Integrity of ETCS messages. Lecture notes in Computer Science, 7865, 2013, pp. 211-222.

[10] D.J. Strom \& P.S. Stansbury. Determining parameters of Lognormal distributions form minimal information. AIHAJ - American Industrial Hygiene Association, 61:6, pp. 877 - 880.

[11] http://systuf.ifsttar.fr/index-en.php.

[12] T. Xu \& T. Tang. The modeling and analysis of Data Communication system (DSC) in Communication Based Train Control (CBTC) with Colored Petri Nets. 8-th International Symposium on Autonomous Decentralized Systems 2007, pp. 83-92.

[13] H. Wang \& S. Liu. Modelling Communication-Based Train Control System: A Case study. 2nd International conference on Industrial Mechatronics and Automation, pp. 453 - 456.

[14] L. Zhang, T. Okamawari, T. Fujii. Performance evaluation of End-toEnd Communication Quality of LTE. Vehicular Technology Conference (VTC Spring), 2012, pp.1-5.

[15] H. Zhao, T. Xu \& T. Tang, Towards modelling and evaluation of availability of communication based train control system. Proceedings of ICCTA. 2009, pp. 860-863.

[16] A. Zimmermann, G. Hommel. Towards modeling and evaluation of ETCS real-time communication and operation, The journal of Systems and Software 77, 2005, pp. 47-54. 